\title{
Desestabilización de emulsiones aceite-agua preparadas con proteínas de soja
}

\author{
Abirached, C. (1)*, Medrano, A. (1), Panizzolo, L. A. (1), Moyna, P. (1) y Añón, M. C. (2) \\ (1) Departamento de Ciencia y Tecnología de los Alimentos. Facultad de Química, Universidad de la República, Uruguay \\ (2) CIDCA-Universidad Nacional de La Plata, La Plata, Buenos Aires, Argentina
}

Contacto: abirached@fq.edu.uy

Recibido: 30/06/2011 - Aprobado: 13/10/2011

\begin{abstract}
$\underline{\text { Resumen }}$
En este trabajo se realizó un estudio de las propiedades emulsionantes de las proteínas de soja glicinina y $\beta$-conglicinina en comparación con el aislado proteico de soja. Para esto se aislaron las fracciones por precipitación isoeléctrica, se les determinó la solubilidad, hidrofobicidad superficial y comportamiento térmico. Se estudió la estabilidad frente al cremado de las emulsiones por medio de un analizador óptico vertical y se establecieron dos constantes de desestabilización correspondientes a los tamaños de partícula observados con un analizador de partículas, mediante una ecuación de segundo orden bifásica (Panizzolo, 2005). Se determinó que las emulsiones preparadas con glicinina son las más inestables en cuanto al cremado y que las preparadas con $\beta$-conglicinina presentan un comportamiento similar al aislado proteico de soja.

Palabras Claves: Cremado, glicinina, $\beta$-conglicinina, aislado proteico de soja.
\end{abstract}

\begin{abstract}
$\underline{\text { Abstract }}$
In this paper a study of emulsifying properties of soy proteins, glycinin and $\beta$-conglycinin, compared with soy protein isolate was conducted. For this, the fractions were isolated by isoelectric precipitation and were examined for solubility, surface hydrophobicity and thermal behaviour. The stability against creaming of emulsions was studied by a vertical optical analyzer, determining two constants of destabilization for the particle sizes observed with a particle analyzer, based on a biphasic second order equation (Panizzolo, 2005). It was determined that the emulsions prepared with glycinin are the most unstable in terms of creaming. The prepared with $\beta$-conglycinin presents a soy protein isolate-like behaviour.

Keywords: Creaming, glycinin, $\beta$-conglycinin, soy protein isolate.
\end{abstract}

\section{Introducción}

Muchas proteínas son moléculas con actividad interfacial que pueden ser utilizadas como emulsionantes por su habilidad de facilitar la formación, mejorar la estabilidad y producir propiedades fisicoquímicas deseadas en las emulsiones de aceite en agua (McClements, 2004)

El estudio de proteínas vegetales, como las proteínas de soja, resulta de interés tecnológico debido a la posibilidad de emplearlas como sustitutos de proteínas de origen animal para su uso en emulsiones alimentarias, de mayor costo. En Uruguay, así como en toda la región, el área sembrada y la producción de soja han tenido un crecimiento vertiginoso. Esta soja es utilizada por la industria aceitera y del biodiesel. Es más, para atender el compromiso de mezcla de $2 \%$ de biodiesel en el gasoil establecido en el marco de la Ley 18.195 se generarán grandes volúmenes de harinas oleaginosas.

El aislado proteico de soja está constituido principalmente por las proteínas glicinina y $\beta$-conglicinina.

El modelo estructural más aceptado de glicinina es un hexámero constituido por seis subunidades monoméricas que tienen la estructura general $\mathrm{AB}$, donde $\mathrm{A}$ representa un polipéptido ácido y $\mathrm{B}$ un polipéptido básico. Las cadenas A y B están unidas por un único puente disulfuro. La glicinina (11S) nativa se compone de seis subunidades ácidas (A) y seis subunidades básicas $(B)\left((A B)_{6}\right)$. Las subunidades $A B$ están asociadas en dos anillos hexagonales que forman un cilindro hueco que se mantiene unido por interacciones hidrofílicas (interacciones electrostáticas / puentes de hidrógeno). Según la solubilidad, el pH y la fuerza iónica, la glicinina (11S) se disocia en la forma $7 \mathrm{~S}\left((\mathrm{AB})_{3}\right)$ y/o en la forma $3 \mathrm{~S}(\mathrm{AB})$. La disociación se debe fundamentalmente a la repulsión electrostática. También puede ocurrir la asociación en la forma $15 \mathrm{~S}$ (dímero de 11S). Diferentes cantidades de las formas $3 \mathrm{~S}, 7 \mathrm{~S}$ y $11 \mathrm{~S}$ están presentes a diferentes $\mathrm{pH}$; a $\mathrm{pH}$ mayores a 6,7 la glicinina está principalmente bajo la forma 11S (Martin et al., 2002).

La $\beta$-conglicinina es una glicoproteína que contiene en torno al $5 \%$ de glúcidos (manosa y N-acetil-glucosamina). Está compuesta por 3 subunidades $\alpha, \alpha^{\prime}$ y $\beta$, de carácter ácido. La estructura completa trimérica se estabiliza mediante interacciones hidrofóbicas. Con $\mathrm{pH}$ comprendidos entre 5 y 10 , cuando la fuerza iónica es baja (aproximadamente 0,1) se forma un dímero de 6 subunidades (9S) (Cheftel et al., 1989).

En este trabajo se estudió las propiedades emulsionantes de estas proteínas en comparación con el aislado proteico de soja. 


\section{Materiales y Métodos}

El aislado proteico de soja (APS), la $\beta$-conglicinina (7S) y la glicinina (11S) se obtuvieron a partir de harina de soja desengrasada por precipitación isoeléctrica (Petruccelli y Añón, 1994; Nagano et al., 1992). Se dispersaron en medio alcalino ( $\mathrm{pH} 8,0)$ y se secaron por liofilización en un liofilizador Christ Alpha 1-4, European model LOC-1M.

Se determinó la solubilidad de las diferentes muestras dispersándolas en solución de fosfato de sodio $10 \mathrm{mM} \mathrm{pH} \mathrm{8,0} \mathrm{al}$ $0,1 \% \mathrm{p} / \mathrm{v}$ durante 60 minutos a temperatura ambiente con agitación constante. Posteriormente, las muestras fueron centrifugadas a $10.000 \mathrm{xg}$ durante 10 minutos a $4{ }^{\circ} \mathrm{C}$. El contenido de proteína en el sobrenadante se determinó utilizando el método de Lowry (Lowry et al., 1951).

Los valores de hidrofobicidad aromática superficial $\left(\mathrm{H}_{0}\right)$ de cada muestra fueron determinados por medio de la sonda fluorescente ANS (ácido 8-anilino 1 naftalen-sulfónico) de acuerdo al método de Hayakawa y Nakai (1985). La hidrofobicidad superficial se determinó como la pendiente inicial de la curva de intensidad de fluorescencia relativa porcentual versus la concentración de proteína según Kato y Nakai (1980).

Se realizaron ensayos de calorimetría diferencial de barrido (DSC) con un calorímetro de barrido diferencial DSC Serie Q100 TA Instruments con software Universal Analysis 2000; se determinaron temperatura y entalpía de desnaturalización (Medrano et al., 2009).

Se estudiaron emulsiones de aceite en agua usando una proporción aceite:agua de 25:75 (v/v).

La fase acuosa fue una dispersión proteica de $1,0 \mathrm{mg} / \mathrm{ml}$ en buffer fosfato de sodio $10 \mathrm{mM}, \mathrm{pH} 7,0$, fuerza iónica 0,02 . Las emulsiones se prepararon a temperatura ambiente, con un homogenizador Ultraturrax T25 (IKA-Labortechnik), utilizando el accesorio dispersante S25 N-10 G a una velocidad de $20.000 \mathrm{rpm}$ durante un minuto. Se les analizó la estabilidad frente al cremado mediante un analizador óptico vertical (Turbiscan Classic MA2000). Las emulsiones se colocaron en una celda cilíndrica de vidrio $(80 \mathrm{~mm})$ para registrar el perfil de retrodispersión (RD) en función de la altura en la celda. Se realizaron medidas cada un minuto durante un período de 60 minutos.

Los datos de RD a una altura del tubo de $10 \mathrm{~mm}$ se ajustaron a una ecuación de segundo orden bifásica desarrollada por Panizzolo (2005) (ec. 1).

$$
R D(t)=\frac{R D_{1}}{\left(R D_{1} k_{1} t+1\right)}+\frac{R D_{2}}{\left(R D_{2} k_{2} t+1\right)}
$$

Siendo $\mathrm{k}_{1}$ y $\mathrm{k}_{2}$ las constantes de velocidad y $\mathrm{RD}_{1}$ y $\mathrm{RD}_{2}$ los porcentajes de retrodispersión a una altura de $10 \mathrm{~mm}$ del tubo a un tiempo $t$ para cada término de la ecuación $1 . \mathrm{k}_{1} \mathrm{y} \mathrm{RD}_{1}$ corresponden a las gotas de mayor tamaño que son las que creman más rápido y $\mathrm{k}_{2}$ y $\mathrm{RD}_{2}$ corresponden a las gotas más pequeñas, las cuales creman más lentamente según lo predicho por la ley de Stokes.

La distribución de tamaño de partícula se determinó por difracción de luz láser y dispersión de luz polarizada, utilizando un analizador de partículas Coulter Counter Multisizer (Coulter Electronics Ltd.).

El tratamiento estadístico de datos se realizó mediante análisis de la varianza (ANOVA) y prueba de mínimas diferencias significativas (LSD) $\operatorname{con} \alpha=0,05$, por medio del programa Statgraphics plus 7.0.

\section{Resultados y Discusión}

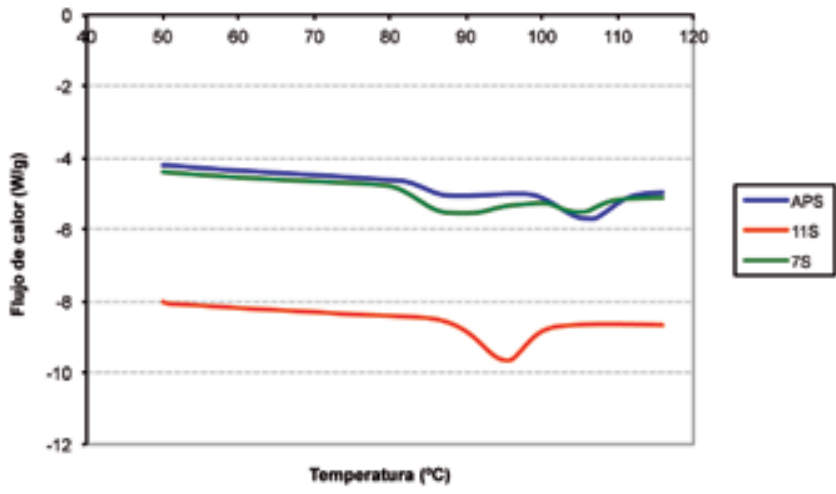

Figura 1. Termogramas de (-) APS, (-) 11S y (-) 7S.

\begin{tabular}{|c|c|c|}
\hline Muestra & Solubilidad (\%) & $\begin{array}{c}\text { Hidrofobicidad } \\
\text { superficial }\end{array}$ \\
\hline 7Sn & $88 \pm 4 \mathrm{a}$ & $14 \pm 6 \mathrm{a}$ \\
$\mathbf{1 1 S n}$ & $88 \pm 6 \mathrm{a}$ & $17 \pm 2 \mathrm{a}$ \\
APSn & $93 \pm 8 \mathrm{a}$ & $30 \pm 5 \mathrm{~b}$ \\
\hline
\end{tabular}

Tabla 1. Hidrofobicidad superficial y solubilidad proteica de las distintas muestras, esta última expresada como: g de proteína soluble en $100 \mathrm{~g}$ de proteína. Los valores que se encuentran en la misma columna con la misma letra no presentan diferencias significativas $(\alpha \leq 0,05)$.

En la Figura 1 se muestra el termograma obtenido por DSC de APS, $11 \mathrm{~S}$ y $7 \mathrm{~S}$. APS presentó dos picos endotérmicos uno a $(86 \pm 10)^{\circ} \mathrm{C}$ y otro a $(105 \pm 2)^{\circ} \mathrm{C}$. El primero de ellos corresponde a la $\beta$-conglicinina y el otro a la glicinina. La fracción 11S presentó un pico endotérmico a $(96 \pm 1){ }^{\circ} \mathrm{C}$. La fracción $7 \mathrm{~S}$ presenta dos picos endotérmicos, uno correspondiente a la $\beta$-conglicinina propiamente dicha $(87 \pm 8){ }^{\circ} \mathrm{C}$ y otro correspondiente a la impurificación con glicinina $(102 \pm 10)^{\circ} \mathrm{C}$, lo cual ya había sido observado por Petruccelli y Añón $(1994,1995)$.

No se encontraron diferencias significativas $(\alpha \leq 0.05)$ en la hidrofobicidad superficial y solubilidad de 7S y $11 \mathrm{~S}$ (Tabla 1).

Las distribuciones de tamaño de partícula expresadas tanto en volumen como en superficie de las emulsiones preparadas exhibieron un carácter bimodal con dos poblaciones de gotas bien definidas: gotas con diámetro mayor y menor a $5 \mu \mathrm{m}$ para $11 \mathrm{~S}$ y mayor y menor a $3 \mu \mathrm{m}$ para 7S y APS (Figura 2). En el rango de partículas de mayor tamaño se distribuye más del $90 \%$ del volumen y en el rango de partículas de menor tamaño se tiene la mayor área y el mayor número de gotas. Este tipo de comportamiento bimodal fue observado con anterioridad por Mitidieri y Wagner (2002) y Panizzolo (2005) y fue atribuido a las condiciones de homogenización. Por lo tanto, al encontrarse dos tamaños de partícula se aplica la ecuación 1 . 


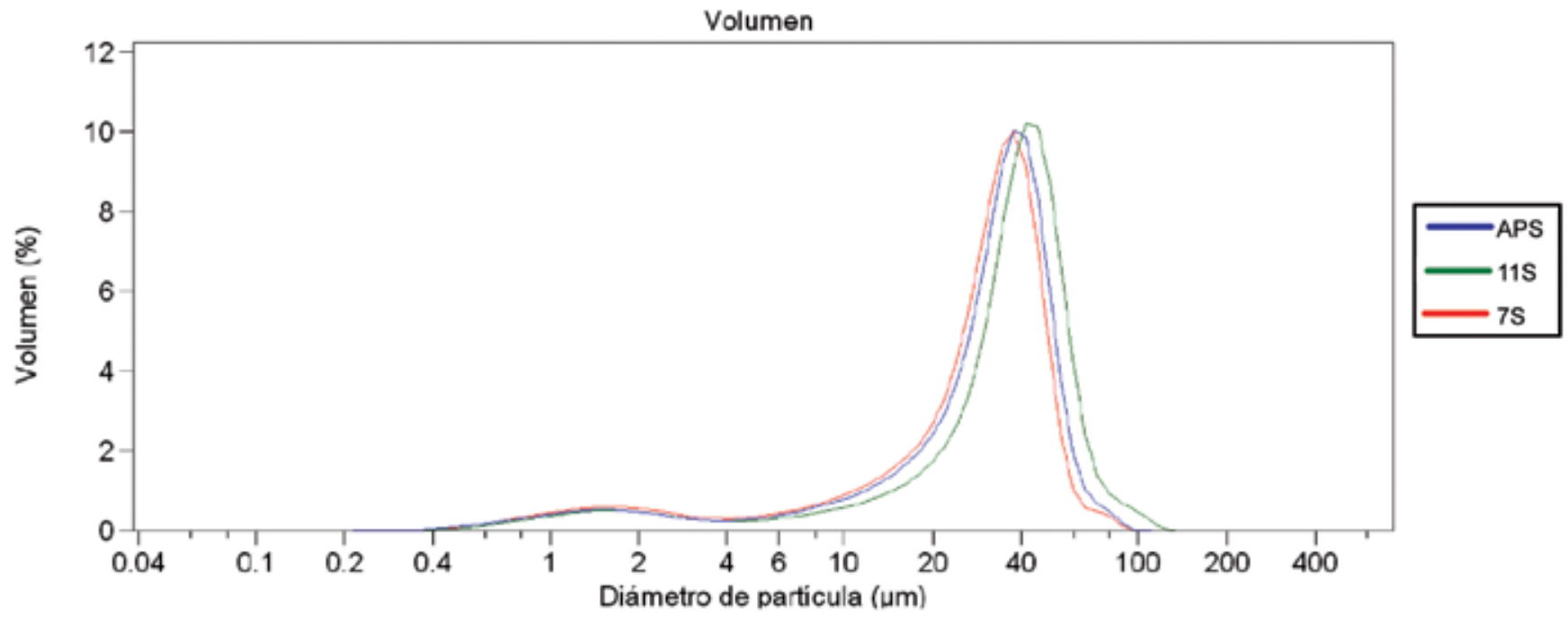

Figura 2. Distribución en volumen de tamaño de gota de las emulsiones preparadas con (—) APS, (一) 11S y (-) 7S.

\begin{tabular}{|c|c|c|c|c|c|}
\hline & $\mathbf{R D}_{\mathbf{0}}$ & $\mathbf{R D}_{\mathbf{1}}$ & $\mathbf{k}_{\mathbf{1}} \times \mathbf{1 0}^{\mathbf{3}}\left(\mathbf{m i n}^{-\mathbf{1}}\right)$ & $\mathbf{R D}_{\mathbf{2}}$ & $\mathbf{k}_{\mathbf{2}} \times \mathbf{1 0}^{\mathbf{4}}\left(\mathbf{m i n}^{-\mathbf{1}}\right)$ \\
\hline $\mathrm{APS}$ & $50 \pm 3 \mathbf{a}$ & $29 \pm 2 \mathbf{a}$ & $9 \pm 1 \mathbf{a}$ & $20 \pm 4 \mathbf{a}$ & $9 \pm 1 \mathbf{a}$ \\
\hline $11 \mathrm{~S}$ & $39 \pm 10 \mathbf{b}$ & $25 \pm 5 \mathbf{b}$ & $16 \pm 9 \mathbf{b}$ & $13 \pm 5 \mathbf{b}$ & $7 \pm 1 \mathbf{b}$ \\
\hline $7 \mathrm{~S}$ & $49 \pm 3 \mathbf{a}$ & $31 \pm 3 \mathbf{a}$ & $10 \pm 1 \mathbf{a}$ & $18 \pm 2 \mathbf{a}$ & $11 \pm 1 \mathbf{c}$ \\
\hline
\end{tabular}

Tabla 2. $\mathrm{RD}_{0}, \mathrm{k}_{1}, \mathrm{RD}_{1}, \mathrm{k}_{2}$ y $\mathrm{RD}_{2}$ para las distintas proteínas. Los valores que se encuentran en la misma columna con la misma letra no presentan diferencias significativas $(\alpha \leq 0,05)$.

El tamaño de las gotas producidas durante la formación de emulsiones depende de dos procesos: 1) la generación de gotas de pequeño tamaño y 2) la rápida estabilización de estas gotas, lo que evita su coalescencia una vez formadas (McClements, 1999). Bajo las mismas condiciones de homogenización se ha demostrado que emulgentes que se adsorben rápidamente producen tamaños de gota más pequeños que otros que se adsorben más lentamente. La tendencia de las gotas a coalescer durante la homogenización depende además de la efectividad de la membrana interfacial a resistir la coalescencia durante la colisión de gotas. Esta resistencia depende de la concentración de moléculas de emulgente presente, así como de su estructura y propiedades fisicoquímicas (dimensión, carga eléctrica, empaquetado, interacciones) (Schubert y Armbruster, 1992).

En general, se puede decir que la mayoría de las moléculas de proteínas adsorbidas son capaces de formar una red en la interfase, propiedad que depende del tipo de proteína usada.

$\mathrm{Al} \mathrm{pH}$ de trabajo $(\mathrm{pH} 7,0)$ las subunidades constituyentes de $7 \mathrm{~S}$ están cargadas negativamente, ya que se está a un $\mathrm{pH}$ mayor que los pI de las mismas (pI $\alpha: 5,2, \mathrm{pI} \alpha^{\prime}: 5,3$, pI $\beta: 5,8-6,2$ ). En cambio, las subunidades A y B (pIA: 4,5-5,4, pIB: 8,0-8,5) de 11S están cargadas negativa y positivamente, cada una (Cheftel et al., 1989).

Aunque tanto la proteína $7 \mathrm{~S}$ como la $11 \mathrm{~S}$ son capaces de formar una red, la red de $11 \mathrm{~S}$ es probablemente menos fuerte, ya que al $\mathrm{pH}$ de trabajo, según lo descrito por Martin et al. (2002), 11S tiene una estructura compacta (se presenta como un hexámero). La proteína $7 \mathrm{~S}$ al tener alta repulsión electrostática dentro de la molécula, y encontrarse cargada negativamente al $\mathrm{pH}$ de trabajo, tendrá más facilidad para desplegarse durante la adsorción y por lo tanto, más probabilidad de formar enlaces físicos y covalentes intermoleculares. Esto explica que las emulsiones preparadas con $11 \mathrm{~S}$ presenten un mayor tamaño de gota que las preparadas con $7 \mathrm{~S}$.
Además, la carga neta negativa de la proteína $7 \mathrm{~S}$ que se encuentra en la interfase evita la coalescencia de las gotas en las emulsiones preparadas con esta proteína.

Según los valores de $\mathrm{k}_{1}$ presentados en la Tabla 2, las emulsiones más inestables frente al cremado son las preparadas con 11S. Esto es debido a que tienen un mayor tamaño de gota que $7 \mathrm{~S}$.

A partir de los valores de entalpía de desnaturalización obtenidos del termograma de APS (Figura 1) se obtiene que la proporción de proteína $7 \mathrm{~S}$ en el APS es de $(27 \pm 4) \%$ y el de $11 \mathrm{~S}$ es $(73 \pm 4) \%$, calculados según las ecuaciones 1 y 2 . Sin embargo, la proteína que rige el comportamiento interfacial de APS es la $7 \mathrm{~S}$, dado que no se encuentran diferencias significativas entre los valores de $\mathrm{RD}_{0}, \mathrm{k}_{1}, \mathrm{RD}_{1}$ y $\mathrm{RD}_{2}$ de éstas (Tabla 2).

Un $\mathrm{RD}_{0}$ alto implica un mayor número de gotas presente inicialmente y $\mathrm{RD}_{0}=\mathrm{RD}_{1}+\mathrm{RD}_{2}$, por lo cual los valores de $\mathrm{RD}$ concuerdan con lo visto con el análisis de tamaño de partícula, las emulsiones preparadas con $11 \mathrm{~S}$ presentan partículas de mayor diámetro $\mathrm{y}$, por ende. son las que presentan el menor $\mathrm{RD}_{0}$, ya que la fracción volumétrica de aceite se mantiene constante (Tabla 2).

Ninguna de las diferencias encontradas en la estabilidad de las emulsiones puede explicarse por diferencia de solubilidad o hidrofobicidad superficial debido a que, como se mencionó anteriormente, no existen diferencias significativas entre $11 \mathrm{~S}$ y $7 \mathrm{~S}$. 


\section{Conclusiones}

Las emulsiones preparadas con glicinina son las más inestables en cuanto al cremado. Las preparadas con $\beta$-conglicinina presentan un comportamiento similar al aislado proteico de soja.

\section{Reconocimientos}

Los autores agradecen a LATU la beca otorgada por medio del convenio LATU-Facultad de Química y el soporte financiero del Programa de Desarrollo Tecnológico (PDT) del Ministerio de Educación y Cultura, la Comisión Sectorial de Investigación Científica (CSIC) y el Plan de Desarrollo de las Ciencias Básicas (PEDECIBA), Uruguay.

\section{Referencias}

\section{- CHEFTEL, J. C.; CUQ, J. L.; LORIENT, D. Proteinas} alimentarias; bioquimica, propiedades funcionales, valor nutritivo, modificaciones químicas. Zaragoza: Acribia, 1989, ISBN: 84-2000649-1.

- HAYAKAWA, S.; NAKAI, S. Relationships of hydrophobicity and net charge to the solubility of milk and soy proteins. En: Journal of Food Science. 1985, 50(2):486-491.

- KATO, A.; NAKAI, S. Hydrophobicity determined by fluorescence probe method and its correlation with surface properties of proteins. En: Biochimica et Biophysica Acta. 1980, 624(1):13-20.

- LOWRY, Oliver H.; ROSEBROUGH, Nira. J.; FARR, Lewis, A.; RANDALL, Rose J. Protein measurement with the folin phenol reagent. En: Journal of Biological Chemistry. 1951, 193(1):265275.

- MARTIN, A. H.; BOS, M.A.; VAN VLIET, T. Interfacial rheological properties and conformational aspects of soy glicinin at the air/water interface. En: Food Hydrocolloids. 2002, 16(1):63-71.

- McCLEMENTS, D. Protein-stabilized emulsions. En: Current Opinion in Coloids \& Interface Science. 2004, 9(5):305-313.

- McCLEMENTS, D. Food emulsions. Principles, practice and techniques. Nueva York: CRC Press, 1999. ISBN: 0-8493-8008-1.

- MEDRANO, A.; ABIRACHED, C.; PANIZZOLO, L.; MOYNA, P.; AÑÓN, M.C. The effect of glycation on foam and structural properties of $\beta$-lactoglobulin. En: Food Chemistry. 2009,113(1):127-133.

- MITIDIERI, F. E.; WAGNER, J. R. Coalescence of o/w emulsions stabilized by whey and isolate soybean proteins. Influence of thermal denaturation, salt addition and competitive interfacial adsorption. En: Food Research International. 2002, 35(6):547557.

- NAGANO, T.; HIROTSUKA, M.; KOHYAMA, K.; NISHINARI, K. Dynamic viscoelastic study on the gelation of 7S globulin from soybeans. En: Journal of Agricultural and Food Chemistry. 1992, 40(6): 941-944.

- PANIZZOLO, L. A. Modificación de proteinas por vía enzimática. Análisis de la relación estructura-funcionalidad de los productos de hidrólisis. Montevideo: UDELAR. Facultad de Química, 2005. (Tesis de Doctorado).

- PETRUCCELLI, S.; AÑÓN, M. C. The relationship between the method of preparation and the structural and functional properties of soy protein isolates. Part I: Structural and hydration properties. En: Journal of Agriculture and Food Chemistry. 1994, 42:21612169.
- PETRUCCELLI, S.; AÑÓN, M. C. Partial reduction of soy protein isolate disulfide bonds. En: Journal of Agriculture and Food Chemistry. 1995, 43:2001-2006.

- SCHUBERT, H.; ARMBRUSTER, H. Principles of formation and stability of emulsions. En: International Chemical Engineering. 1992, 32(1):14-28. 